\title{
Dynamic DNA shortening by telomere-binding protein Cdc13
}

Yi-Yun Lin ${ }^{1}$, Min-Hsuan Li ${ }^{1}$, Yen-Chan Chang ${ }^{2}$, Peng-Yu Fu ${ }^{1}$, Ryosuke L. Ohniwa ${ }^{3,4}$, Hung-Wen $\mathrm{Li}^{2 *}$, Jing-Jer $\operatorname{Lin}^{1,5 *}$

1. Institute of Biochemistry and Molecular Biology, National Taiwan University, Taiwan

2. Department of Chemistry, National Taiwan University, Taiwan

3. Faculty of Medicine, University of Tsukuba, Japan

4. Center for Biotechnology, National Taiwan University, Taiwan

5. Institute of Biopharmaceutical Sciences, National Yang-Ming University, Taiwan

* To whom correspondence should be sent: jingjerlin@ntu.edu.tw (JJL) or

hwli@ntu.edu.tw (HWL). 


\section{Supplementary Table EV1}

Table EV1. Sequences of DNA substrates used in this study.

DupW

CCTGCTCGCTTCGCTACTTGGAGCCACTATCGACTACGCGATCATGGCGACCACACCCGTCCTGTGGATCCTCTACGCCGGACGCATCGTGGCCGGC ATCACCGGCGCCACAGGTGCGGTTGCTGGCGCCTATATCGCCGACATCACCGATGGGGAAGATCGGGCTCGCCACTTCGGGCTCATGAGCGCTTGTT TCGGCGTGGGTATGGTGGCAGGCCCCGTGGCCGGGGGACTGTTGGGCGCCATCTCCTTGCATGCACCATTCCTTGCGGCGGCGGTGCTCAACGGCCT CAACCTACTACTGGGCTGCTTCCTAATGCAGGAGTCGCAT*AGGGAGAGCGTCGACCGAT - 3'

DupW-TG15

CCTGCTCGCTTCGCTACTTGGAGCCACTATCGACTACGCGATCATGGCGACCACACCCGTCCTGTGGATCCTCTACGCCGGACGCATCGTGGCCGGC ATCACCGGCGCCACAGGTGCGGTTGCTGGCGCCTATATCGCCGACATCACCGATGGGGAAGATCGGGCTCGCCACTTCGGGCTCATGAGCGCTTGTT TCGGCGTGGGTATGGTGGCAGGCCCCGTGGCCGGGGGACTGTTGGGCGCCATCTCCTTGCATGCACCATTCCTTGCGGCGGCGGTGCTCAACGGCCT CAACCTACTACTGGGCTGCTTCCTAATGCAGGAGTCGCAT*AGGGAGAGCGTCGACCGATTGTGGTGTGTGTGGG-3’

DupW-A15

CCTGCTCGCTTCGCTACTTGGAGCCACTATCGACTACGCGATCATGGCGACCACACCCGTCCTGTGGATCCTCTACGCCGGACGCATCGTGGCCGGC ATCACCGGCGCCACAGGTGCGGTTGCTGGCGCCTATATCGCCGACATCACCGATGGGGAAGATCGGGCTCGCCACTTCGGGCTCATGAGCGCTTGTT TCGGCGTGGGTATGGTGGCAGGCCCCGTGGCCGGGGGACTGTTGGGCGCCATCTCCTTGCATGCACCATTCCTTGCGGCGGCGGTGCTCAACGGCCT CAACCTACTACTGGGCTGCTTCCTAATGCAGGAGTCGCAT*AGGGAGAGCGTCGACCGATAAAAAAAAAAAAAAA-3’

\section{DupW-A5TG15}

CCTGCTCGCTTCGCTACTTGGAGCCACTATCGACTACGCGATCATGGCGACCACACCCGTCCTGTGGATCCTCTACGCCGGACGCATCGTGGCCGGC ATCACCGGCGCCACAGGTGCGGTTGCTGGCGCCTATATCGCCGACATCACCGATGGGGAAGATCGGGCTCGCCACTTCGGGCTCATGAGCGCTTGTT TCGGCGTGGGTATGGTGGCAGGCCCCGTGGCCGGGGGACTGTTGGGCGCCATCTCCTTGCATGCACCATTCCTTGCGGCGGCGGTGCTCAACGGCCT CAACCTACTACTGGGCTGCTTCCTAATGCAGGAGTCGCAT*AGGGAGAGCGTCGACCGATAAAAATGTGGTGTGTGTGGG -3’

DupC

ATCGGTCGACGCTCTCCCT*ATGCGACTCCTGCATTAGGAAGCAGCCCAGTAGTAGGTTGAGGCCGTTGAGCACCGCCGCCGCAAGGAATGGTGCAT GCAAGGAGATGGCGCCCAACAGTCCCCCGGCCACGGGGCCTGCCACCATACCCACGCCGAAACAAGCGCTCATGAGCCCGAAGTGGCGAGCCCGATC TTCCCCATCGGTGATGTCGGCGATATAGGCGCCAGCAACCGCACCTGTGGCGCCGGTGATGCCGGCCACGATGCGTCCGGCGTAGAGGATCCACAGG ACGGGTGTGGTCGCCATGATCGCGTAGTCGATAGTGGCTCCAAGTAGCGAAGCGAGCAGG - 3’

\section{DupW2-TG15}

CCAATCAATTCTTGCGGAGAACTGTGAATGCGCAAACCAACCCTTGGCAGAACATATCCATCGCGTCCGCCATCTCCAGCAGCCGCACGCGGCGCAT CTCGGGCAGCGTTGGGTCCTGGCCACGGGTGCGCATGATCGTGCTCCTGTCGTTGAGGACCCGGCTAGGCTGGCGGGGTTGCCTTACTGGTTAGCAG AATGAATCACCGATACGCGAGCGAACGTGAAGCGACTGCTGCTGCAAAACGTCTGCGACCTGAGCAACAACATGAATGGTCTTCGGTTTCCGTGTTT CGTAAAGTCTGGAAACGCGGAAGTCAGCGCCCTGCACCATTATG*TCCGGATCTGCATCGTGTGGTGTGTGTGGG - 3’

DupC2

CGATGCAGATCCGGA*CATAATGGTGCAGGGCGCTGACTTCCGCGTTTCCAGACTTTACGAAACACGGAAACCGAAGACCATTCATGTTGTTGCTCA GGTCGCAGACGTTTTGCAGCAGCAGTCGCTTCACGTTCGCTCGCGTATCGGTGATTCATTCTGCTAACCAGTAAGGCAACCCCGCCAGCCTAGCCGG GTCCTCAACGACAGGAGCACGATCATGCGCACCCGTGGCCAGGACCCAACGCTGCCCGAGATGCGCCGCGTGCGGCTGCTGGAGATGGCGGACGCGA TGGATATGTTCTGCCAAGGGTTGGTTTGCGCATTCACAGTTCTCCGCAAGAATTGATTGG - 3’

\section{Dup593W+TG15}

CGCAGTCAGGCACCGTGTATGAAATCTAACAATGCGCTCATCGTCATCCTCGGCACCGTCACCCTGGATGCTGTAGGCATAGGCTTGGTTATGCCGG TACTGCCGGGCCTCTTGCGGGATATCGTCCATTCCGACAGCATCGCCAGTCACTATGGCGTGCTGCTAGCGCTATATGCGTTGATGCAATTTCTATG CGCACCCGTTCTCGGAGCACTGTCCGACCGCTTTGGCCGCCGCCCAGTCCTGCTCGCTTCGCTACTTGGAGCCACTATCGACTACGCGATCATGGCG ACCACACCCGTCCTGTGGATCCTCTACGCCGGACGCATCGTGGCCGGCATCACCGGCGCCACAGGTGCGGTTGCTGGCGCCTATATCGCCGACATCA CCGATGGGGAAGATCGGGCTCGCCACTTCGGGCTCATGAGCGCTTGTTTCGGCGTGGGTATGGTGGCAGGCCCCGTGGCCGGGGGACTGTTGGGCGC CATCTCCTTGCATGCACCATTCCTTGCGGCGGCGGTGCTCAACGGCCTCAACCTACTACTGGGCTGCTTCCTAATGCAGGAGTCGCAT*AGGGAGAG CGTCGACCGATTGTGGTGTGTGTGGG-3’

\section{Dup593C}

ATCGGTCGACGCTCTCCCT*ATGCGACTCCTGCATTAGGAAGCAGCCCAGTAGTAGGTTGAGGCCGTTGAGCACCGCCGCCGCAAGGAATGGTGCAT GCAAGGAGATGGCGCCCAACAGTCCCCCGGCCACGGGGCCTGCCACCATACCCACGCCGAAACAAGCGCTCATGAGCCCGAAGTGGCGAGCCCGATC TTCCCCATCGGTGATGTCGGCGATATAGGCGCCAGCAACCGCACCTGTGGCGCCGGTGATGCCGGCCACGATGCGTCCGGCGTAGAGGATCCACAGG ACGGGTGTGGTCGCCATGATCGCGTAGTCGATAGTGGCTCCAAGTAGCGAAGCGAGCAGGACTGGGCGGCGGCCAAAGCGGTCGGACAGTGCTCCGA GAACGGGTGCGCATAGAAATTGCATCAACGCATATAGCGCTAGCAGCACGCCATAGTGACTGGCGATGCTGTCGGAATGGACGATATCCCGCAAGAG GCCCGGCAGTACCGGCATAACCAAGCCTATGCCTACAGCATCCAGGGTGACGGTGCCGAGGATGACGATGAGCGCATTGTTAGATTTCATACACGGT GCCTGACTGCG-3'

Biotin-CA15

Biotin-CCCACACACACCACA-3,

R5TG40

GCGTAGGGTGTGGTGTGTGTGGGTGTGGTGTGTGTGGGTGTGGTG

* abasic site 


\section{Supplementary Table EV2}

Table EV2

\begin{tabular}{cccr} 
Cdc13 (nM) & $\begin{array}{c}\text { Fraction folded } \\
\text { DNA }(\theta)\end{array}$ & $\begin{array}{c}\text { Fraction non- } \\
\text { folded DNA }(1-\theta)\end{array}$ & \multicolumn{1}{c}{$\mathrm{n}$} \\
\hline 4 & 0.12 & 0.88 & 76 \\
8 & 0.12 & 0.88 & 135 \\
15 & 0.18 & 0.82 & 81 \\
30 & 0.33 & 0.67 & 105 \\
60 & 0.66 & 0.34 & 70 \\
\hline
\end{tabular}


Supplementary Table EV3

Table EV3

DNA Length

\begin{tabular}{lrccc}
\cline { 2 - 5 } & Peak & AFM (nm) & $\begin{array}{c}\text { Step Size } \\
(\mathrm{nm})^{1}\end{array}$ & $\begin{array}{c}\text { Calculated DNA } \\
\text { length (bp) }\end{array}$ \\
\hline DNA alone (L1) & & 192.5 & & 593 \\
Cdc13-DNA complex (L3) & 1 & 85.9 & & \\
& 2 & 61.3 & 24.6 & 76 \\
& 3 & 38.6 & 22.7 & 70 \\
\hline
\end{tabular}

1 Step size $=\operatorname{Peak}(n)-\operatorname{Peak}(n+1)$

${ }^{2}$ Calculated DNA length $(\mathrm{bp})=$ [DNA length $\left.(\mathrm{nm}) / 3.4(\mathrm{~nm})\right] \times 10.5$ 
Supplementary Fig. EV1

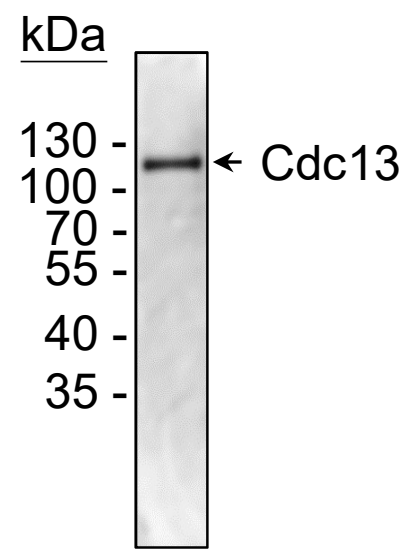

Fig. EV1. Purification of $\mathrm{Cdc} 13$. Cdc13 with $6 \mathrm{xHis}$ tag was purified from sf21 using a Ni-NTA-agarose resin. A Coomassie Blue-stained 10\% SDS-polyacrylamide gel of $1 \mu \mathrm{g}$ each of purified $\mathrm{Cdc} 13 \mathrm{p}$ is presented. 

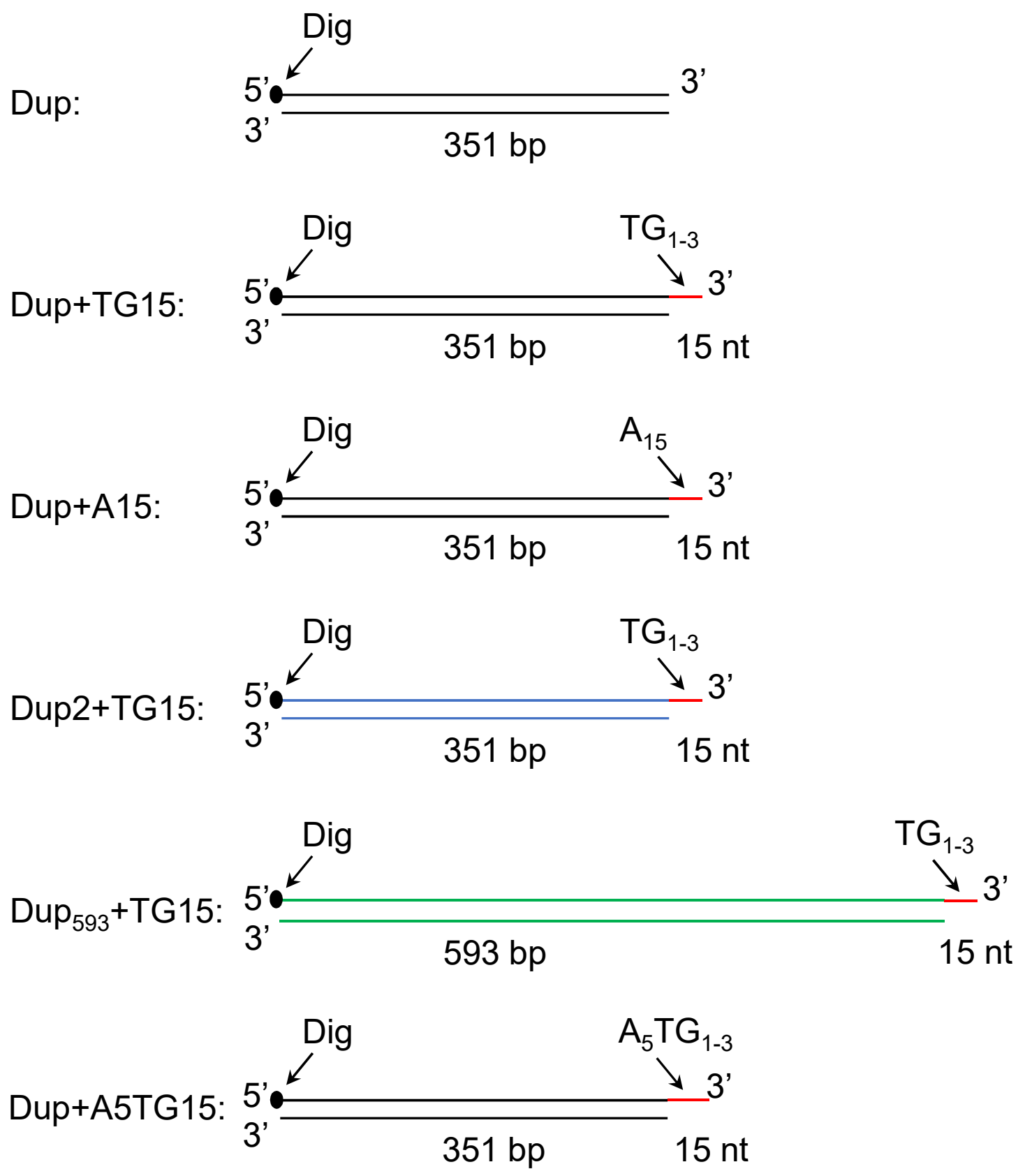

Fig. EV2. Schematic presentation of DNA substrates used in TPM experiments. 


\section{Supplementary Fig. EV3}

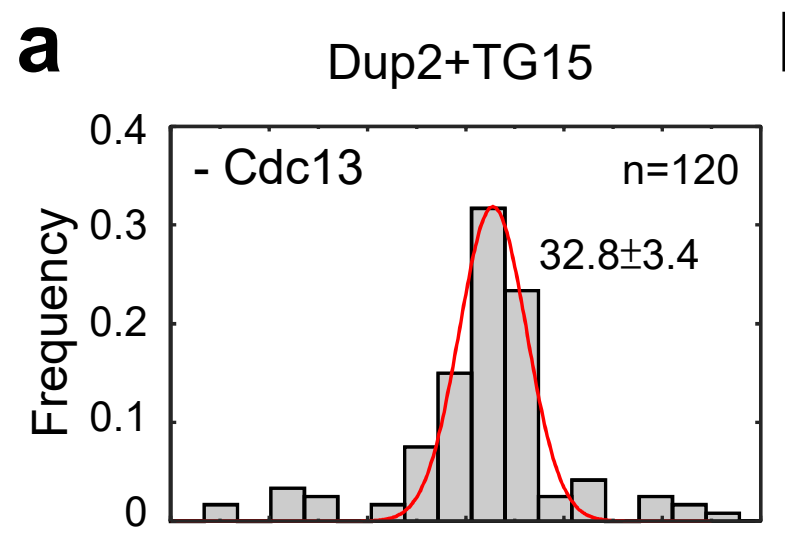

b Dup $_{593}+\mathrm{TG} 15$
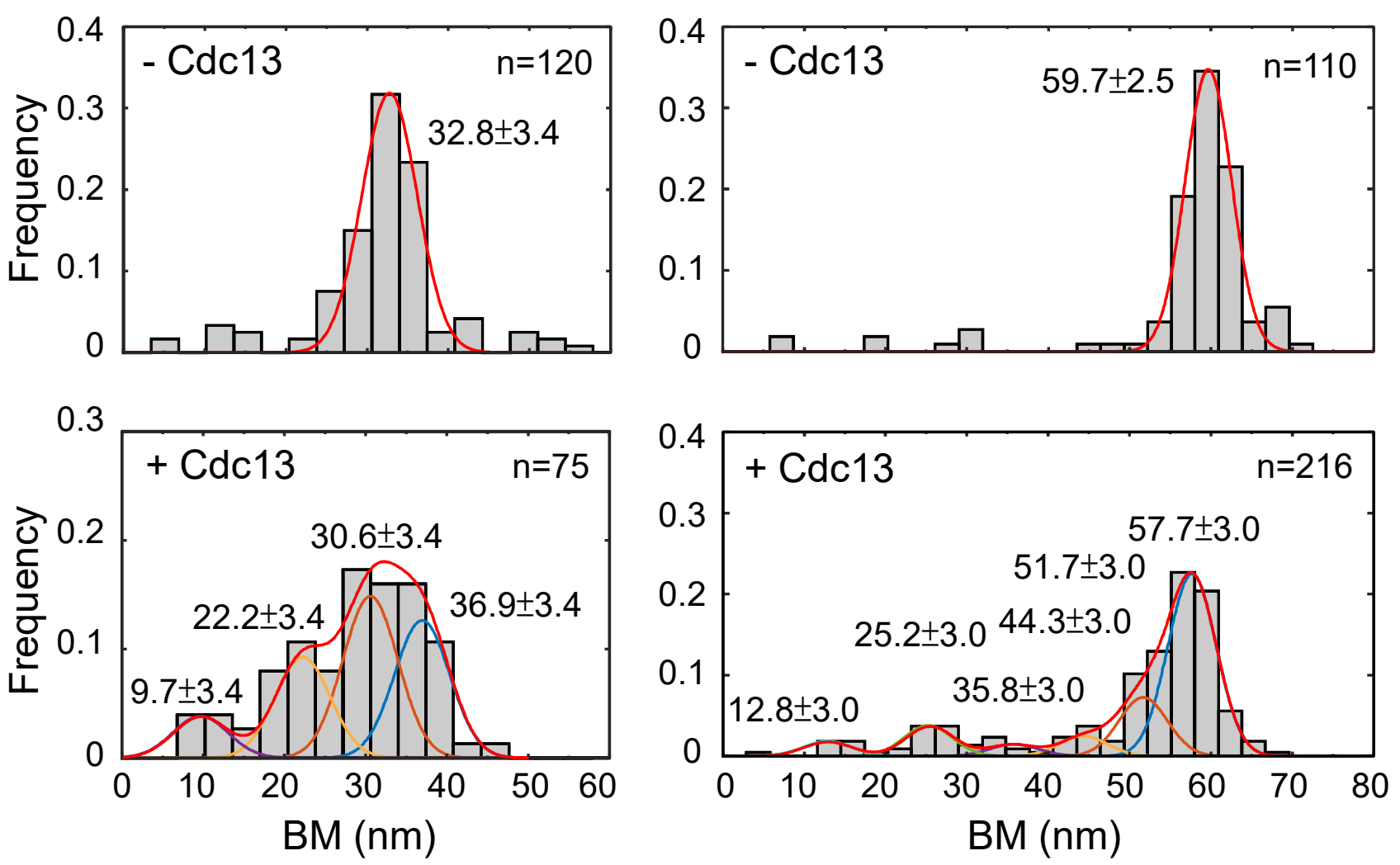

Fig. EV3. TPM analysis of Cdc13 binding to Dup2+TG15 and Dup ${ }_{593}+$ TG15. (a) Cdc13 at $15 \mathrm{nM}$ was incubated with the Dup2+TG15 tethered beads at $25^{\circ} \mathrm{C}$ for $5 \mathrm{~min}$. The BM histograms of DNA substrate in the absence (top) or in the presence of Cdc13 (bottom) were determined. (b) As above, the Dup $_{593}+$ TG15 DNA was used as the substrate. 


\section{Supplementary Fig. EV4}

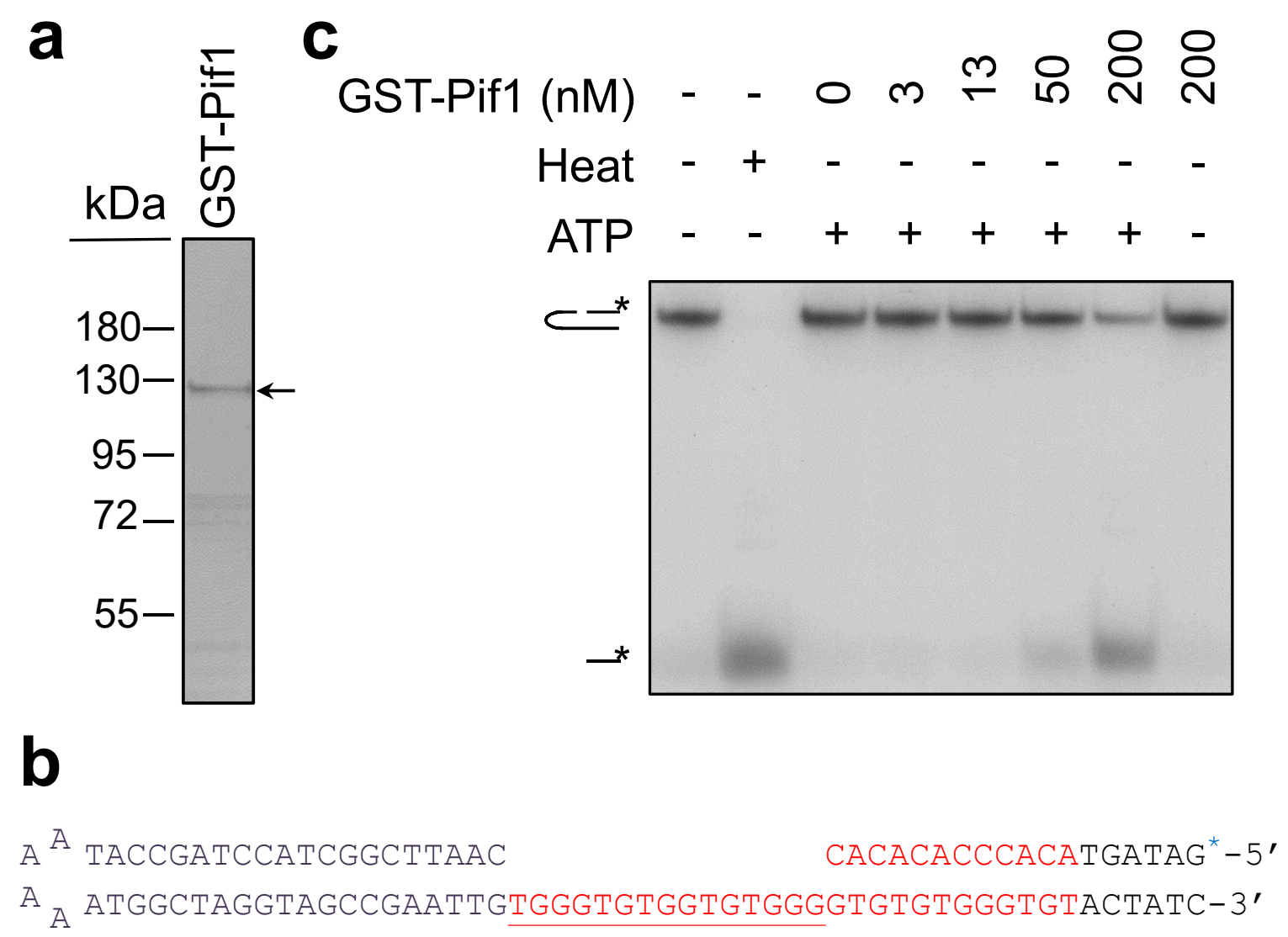

Fig. EV4. Purification and activity analysis of GST-Pif1 protein. (a) Purification of wild-type GST-Pif1. The glutathione S-transferases (GST) tagged Pif1, GST-Pif1, was expressed and purified from E. coli BL21 cells using glutathione agarose followed by Bio-Rex 70 ion exchange resin. One $\mu \mathrm{g}$ of the purified protein was separated by $10 \%$ SDS-polyacrylamide gel. (b) DNA substrate used in helicase activity assay. Telomeric DNA sequences were marked in red. The position of ${ }^{32} \mathrm{P}-$ labeled (blue) oligonucleotide was indicated. (c) Helicase activity analysis of the purified GST-Pif1. The ${ }^{32} \mathrm{P}-$ labeled duplex gapped-DNA substrates were incubated with varying concentration $(0,3,13,50,200 \mathrm{nM})$ of Pif1 in the presence $(+)$ or absence (-) of ATP at $30^{\circ} \mathrm{C}$ for $10 \mathrm{~min}$. The reaction products were separated by $6 \%$ native polyacrylamide gels. The autoradiograph of the separated reaction products is presented. 